\title{
Reducing Frustration and Improving Management of Chronic Pain in Primary Care: Is Shared Decision-making Sufficient?
}

\author{
Marianne S. Matthias, $P h D^{7,2,3}$ and Stephen G. Henry, MD ${ }^{4,5}$ \\ 'VA HSR\&D Center for Health Information and Communication, Roudebush VA Medical Center, Indianapolis, IN, USA; ${ }^{2}$ Department of Medicine, \\ Indiana University School of Medicine, Indianapolis, IN, USA; ${ }^{3}$ Regenstrief Institute, Indianapolis, IN, USA; ${ }^{4}$ Department of Internal Medicine, University \\ of California, Davis, Sacramento, CA, USA; ${ }^{5}$ C Davis Center for Healthcare Policy and Research, Sacramento, CA, USA.
}

J Gen Intern Med 37(1):227-8

DOI: $10.1007 / \mathrm{s} 11606-021-06967-3$

(c) This is a U.S. government work and not under copyright protection in the

U.S.; foreign copyright protection may apply 2021

$\mathrm{D}$ espite long-standing efforts to promote productive discussions about chronic pain treatment, these conversations are often still frustrating for both patients and clinicians. These frustrations can be exacerbated when opioids are involved. Shared decision-making, a process in which patients and clinicians share opinions, information, and preferences in an effort to reach mutual agreement on a treatment, ${ }^{1}$ is a wellrecognized strategy for fostering productive treatment discussions. Policymakers, patient advocates, and medical ethicists have broadly promoted shared decision-making. Consistent with this stance, federal guidelines emphasize the need to involve patients in treatment decisions about chronic pain. Definitions of shared decision-making vary, but the core elements, as defined by the classic Charles et al. model, are 1) at least two parties are involved; 2) both parties participate in decision-making; 3) participants share information, including discussion of treatment options and their potential consequences (e.g., risks/benefits); and 4) both parties agree on the final decision. ${ }^{1}$

Most chronic pain is managed in primary care, so we focus our comments on treatment discussions between primary care clinicians (i.e., primary care physicians, nurse practitioners, physician assistants) and their patients. Ensuring that patients are engaged in decisions about their care is important; however, in this essay, we argue that for shared decision-making to take place, it must arise from a foundation of a strong, trusting patient-clinician relationship. Without this foundation, engaging in honest sharing of information, opinions, and preferences is more challenging - especially in pain care where mutual frustration regarding pain treatment and opioid prescribing is

Received March 18, 2021

Accepted June 4, 2021

Published online June 25, 2021 common. Fostering a productive relationship involves taking steps to ensure the patient feels believed and cared for, using behaviors such as listening to and validating the patient's pain experience, expressing empathy, and demonstrating concern for the patient. Creating and maintaining this therapeutic atmosphere then set the stage for productive, shared discussions about pain treatment.

Several aspects of chronic pain treatment fit well within the shared decision-making model. Chronic pain treatments are preference-sensitive, meaning that, while numerous treatments exist, there is rarely one clear best choice. In addition, many behavioral and nonpharmacologic pain treatments require active patient participation to achieve results, and patients are more likely to follow through with a treatment when they participate in decision-making. ${ }^{2}$ However, traditional models of shared decision-making fall short in a number of ways. First, to engage in shared decision-making, patients and clinicians must agree on the clinical problem. But patients and clinicians often disagree about the nature and meaning of chronic pain, especially when there is no clear precipitating injury or pain seems out of proportion with findings from imaging or the physical exam. Patients tend to understand chronic pain through a biological lens, whereas clinicians tend to view pain as a biopsychosocial phenomenon. For example, a patient might believe that worsening low-back pain represents a new injury that warrants imaging, while the clinician attributes the pain to deconditioning or psychosocial stress. Without a shared understanding of the problem, patients and clinicians are less likely to agree about both treatment goals and what treatment options are reasonable.

Second, most chronic pain treatments have small to moderate effects, so patients typically need to combine multiple treatments and modalities (e.g., pharmacologic, movementbased, behavioral, and integrative therapies) to achieve optimal pain control. ${ }^{3}$ Because of this, treating pain is complex, with an almost infinite number of treatment combinations that require trial and error. As a result, it is impractical to discuss and elicit patient preferences for every reasonable combination. Instead, decision-making in chronic pain is an iterative process whereby patients and clinicians continually reassess the problem and adjust the treatment plan over time without 
always formally engaging in elements of shared decisionmaking such as explicit discussions of preferences, risks, and benefits.

Finally, shared decision-making models do not fully account for disagreement about treatment options, nor do they suggest strategies for managing disagreement when it arises. Although some shared decision-making models acknowledge a role for persuasion, ${ }^{4}$ they are based on a transactional view of communication in which patients and clinicians engage in a rational exchange of information and opinions. The 2016 CDC guidelines for opioid prescribing reflect this transactional perspective by emphasizing information exchange about risks and benefits of treatment options. Despite the importance of such discussions, these models do not offer strategies for navigating disagreements. Disagreements may occur throughout the course of diagnosing and treating pain; however, they are more likely to occur when opioids are discussed, which can lead to emotionally charged discussions and mutual distrust. ${ }^{5}$ Such breakdowns can have potentially deleterious consequences: A recent study ${ }^{6}$ found that opioid tapering was associated with patient termination of care, which can have far-reaching negative consequences for patients.

A key conclusion that follows from these points is that, while shared decision-making is an important approach to treatment decision-making and patient-centered care, it is often insufficient for achieving optimal pain care - particularly when disagreements about opioids occur. We are not arguing that shared decision-making is inappropriate for chronic pain treatment. Our point is that a narrow focus on shared decisionmaking and the steps involved (e.g., discussing alternatives, pros/cons, risks/benefits) has the potential to obscure the broader need of creating and maintaining an atmosphere of care, concern, and mutual trust, which patients have identified as integral to effective pain care. ${ }^{5}$ For these reasons, unless patients are at imminent risk of serious harm, clinicians' first priority should be improving or maintaining the quality of the patient-clinician relationship when managing chronic pain. Relationship-building behaviors often overlap with elements of shared decision-making (e.g., listening to patients' opinions and eliciting their desires), but also include additional behaviors such as acknowledging patients' fears about uncontrolled pain, taking a patient's pain seriously, and convincing patients that clinicians are seeking to serve patients' best interests rather than merely to comply with clinic policies. These elements are particularly important when opioids are part of the discussion, given the current environment that encourages clinicians to limit and taper opioid prescriptions.

Placing greater emphasis on the patient-clinician relationship better reflects the clinical realities of pain management and helps to overcome some shortcomings of shared decisionmaking in chronic pain management. Engaging in behaviors that are part of the process of shared decision-making, such as sharing opinions and listening to one another, can go a long way toward relationship building and thus may render the desired outcome of shared decision-making (mutual agreement ${ }^{1}$ ) less important. In other words, if a patient trusts their clinician and is confident that he or she is committed to their best interests, the patient is more likely to be open to trying a different treatment or a lower opioid dose, even if they are initially skeptical. ${ }^{7}$ Finally, creating an environment of care, concern, and mutual respect has its own therapeutic value that can be as important as the treatment itself.

Chronic pain care has long been characterized by mutual frustration, as patients struggle to feel believed and clinicians struggle to find safe and effective pain treatments. Shared decision-making is an important approach that can help create a collaborative atmosphere in which patients and clinicians work toward the same goal. However, clinicians should not lose sight of the more important and fundamental task of building and nurturing the clinician-patient relationship. It is from this solid foundation that effective, mutually satisfying pain care, including shared decision-making, can best occur.

Acknowledgements: The authors would like to thank Matthew Bair, M.D., M.S., and Richard L. Kravitz, M.D., M.S.P.H., for their comments on an earlier draft of this manuscript.

Corresponding Author: Marianne S. Matthias, PhD; VA HSR\&D Center for Health Information and Communication, Roudebush VA Medical Center, Indianapolis, IN, USA (e-mail: mmatthia@iu.edu).

\section{REFERENCES}

1. Charles C, Gafni A, Whelan T. Shared Decision-making in the Medical Encounter: What Does It Mean? (or It Takes at Least Two to Tango). Soc Sci Med 1997;44(5):681-692. DOI: https://doi.org/10.1016/S0277-9536(96) 00221-3

2. Joosten EA, DeFuentes-Merrilas L, de Weert GH, Sensky T, van der Staak CP, de Jong CA. Systematic review of the effects of shared decisionmaking on patient satisfaction, treatment adherence, and health status. Psychother Psychosom 2008;77(4):219-226.

3. Kerns RD, Krebs EE, Atkins D. Making integrated multimodal pain care a reality: a path forward. J Gen Intern Med 2018;33:1-3.

4. Makoul G, Clayman ML. An Integrative Model of Shared Decision Making in Medical Encounters. Patient Educ Couns 2006;60(3):301-312. doi: https://doi.org/10.1016/j.pec.2005.06.010

5. Henry SG, Matthias MS. Patient-Clinician Communication About Pain: a Narrative Review and Conceptual Model. Pain Med 2018;19(11):2154-65. DOI: https://doi.org/10.1093/pm/pny003

6. Perez HR, Buonora M, Cunningham C, Moonseong H, Starrels $\mathbf{J L}$. Opioid taper is associated with subsequent termination of care: a retrospective cohort study. J Gen Intern Med 2020;35:36-42.

7. Matthias MS, Talib TL, Huffman MA. Managing chronic pain in an opioid crisis: What is the role of shared decision-making? Health Commun 2020;35(10): 1239-47.

Publisher's Note: Springer Nature remains neutral with regard to jurisdictional claims in published maps and institutional affiliations. 